\section{Case Report} Journal of Epilepsy Research pISSN 2233-6249 / elSSN 2233-6257

Received April 21, 2021

Revised July 12, 2021

Accepted August 27, 2021

Corresponding author:

Sachin Sureshbabu, DM

Department of Neurosciences, Aster

Malabar Institute of Medical Sciences

Hospital, Kozhikode 673016, India

Tel. $+91-920-702-8028$

Fax. +91-495-274-1329

E-mail; drsachins1@rediffmail.com

\title{
Therapeutic Challenge in a Case of Recent Onset Refractory Cluster Seizures
}

Sachin Sureshbabu, DM ${ }^{1}$, Muralikrishnan Veleri Padmanabhan, MCh ${ }^{1}$, Jacob Alappat, MCh', Smilu Mohanlal, MD' ${ }^{1}$, Sujith Janardhanan, $\mathrm{MD}^{2}$, Sellam Karunanidhi, $\mathrm{MD}^{3}$, Lakshminarayan Kannan, DM ${ }^{4}$, Dinesh Nayak, DM ${ }^{4}$, Biju Shekhar, MD ${ }^{5}$

${ }^{1}$ Department of Neurology, ${ }^{2}$ Department of Radiology, ${ }^{3}$ Department of Nuclear Medicine, Aster Malabar Institute of Medical Sciences Hospital, Kozhikode; ${ }^{4}$ Department of Neurology, Glaenagles Global Hospital, Chennai; ${ }^{5}$ Department of Neuroanaesthesia, Aster Malabar Institute of Medical Sciences Hospital, Kozhikode, India

A dilemma exists in context to the timing of surgery in a case presenting with explosive onset seizures secondary to a focal cortical dysplasia (FCD). This case report highlights the challenges faced in the management of a 4-year-old child with recent onset cluster seizures refractory to anti-epileptic drugs. A 4-year-old girl presented with an acute onset of cluster seizures (up to 32 in a day), semiologically characterized by tonic upper limb extension and laughter lasting for few seconds with no response to multiple anti-epileptic drugs. The clinical, electrographic, neuroimaging and interictal positron emission tomography data were concordant and consistent with a left middle frontal gyrus dysplasia which was successfully resected under electrocorticographic guidance. Patient is seizure free at 2 months of follow up. (Engel Class 1). Surgical resection is feasible and potentially more effective in the early phase of clinical presentation of FCD. (2021;11:146-149)

Key words: Drug resistant epilepsy, Focal cortical dysplasia, Electrocorticography

\section{Introduction}

Epilepsy surgery is the treatment of choice for drug resistant epilepsy, but to determine the timing of surgery is challenging and is case based. Focal cortical dysplasia type $2 b$ (FCD type $2 b$ ) has the best prognosis in terms of seizure freedom post-surgery; however, there is minimal literature available on the treatment of explosive onset FCD. We report a 4-year-old girl who presented with explosive onset FCD and achieved complete seizure freedom by electrocorticography (ECoG) guided focal resection.

\section{Case Report}

A 4-year-old right-handed girl born to unrelated parents was referred to our facility for unremitting cluster seizures since past 2 weeks. The description given by parents was of brief events lasting approximately 10 seconds in which the child had up rolling of eyeballs followed by tonic extension of both the hands forward and giggling. The frequency of seizures varied from 20-30/day and there was no past history of exanthematous illness, developmental delay, febrile seiz- ures, head trauma, and perinatal insult. General physical examination, systemic and neurological examination were unremarkable. She was evaluated with video electroencephalogram (EEG) for recording of events. The interictal record (IED) showed spike and wave discharges along with rhythmic fast activity localized predominantly to the left frontal region (F3>Fz>Fp1). Four habitual clinical events were recorded along with two electrographic seizures (Fig. 1). The semiology was similar to that described in the history. The early recovery of the left upper limb from the tonic posture helped us lateralize the onset to left hemisphere. Vocalization was present before giggling and smiling. The seizure pattern analysis along with the IEDs suggested a spread of seizure from the posterior frontal/premotor region, (which accounts for the elementary motor automatisms) towards the mesial aspect, ultimately resulting in laughter. Based on this electroclinical hypothesis, magnetic resonance imaging (MRI) brain epilepsy protocol was done. MRI showed abnormal gyral pattern with cortical thickening and blurring of grey white matter junction in the left middle frontal gyrus. An interictal positron emission tomography revealed further confirmatory evidence in the form of left anterior frontal hypometabolism (Fig. 2).

The child's seizures were not controlled by multiple anti-epileptic 
A

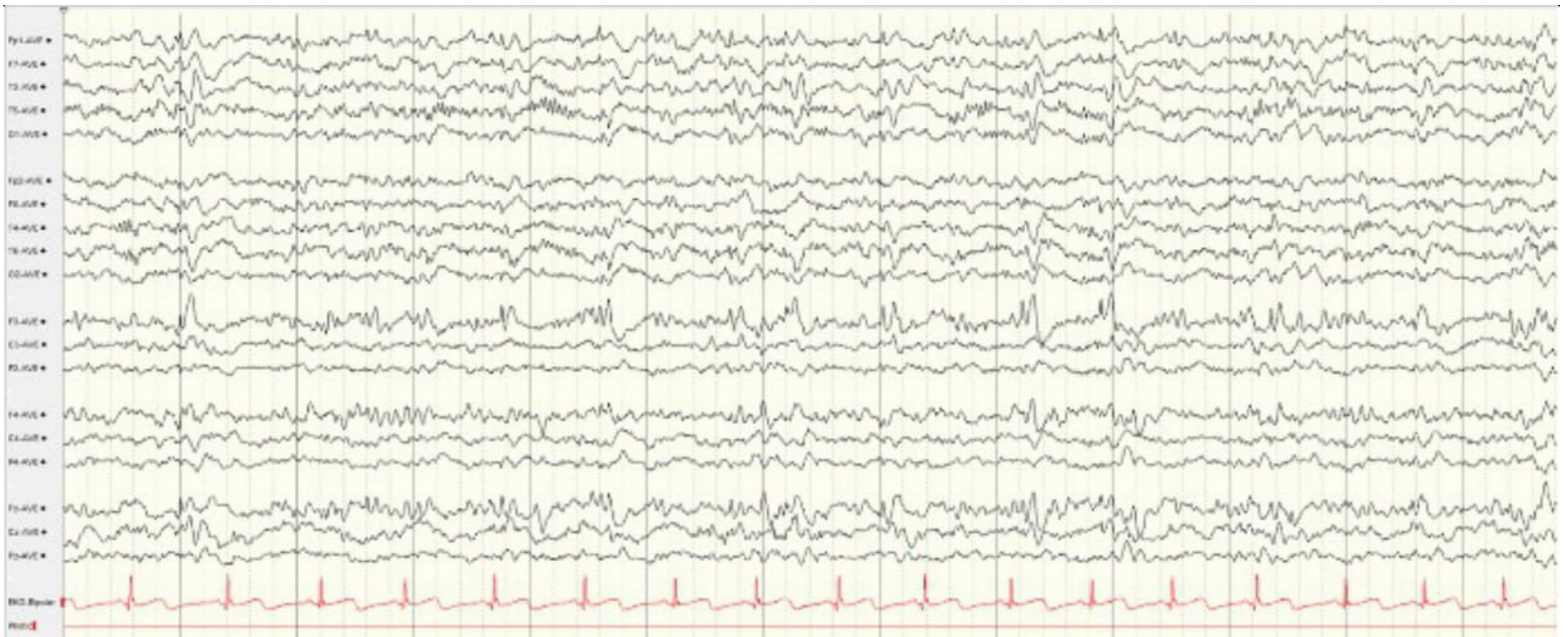

B

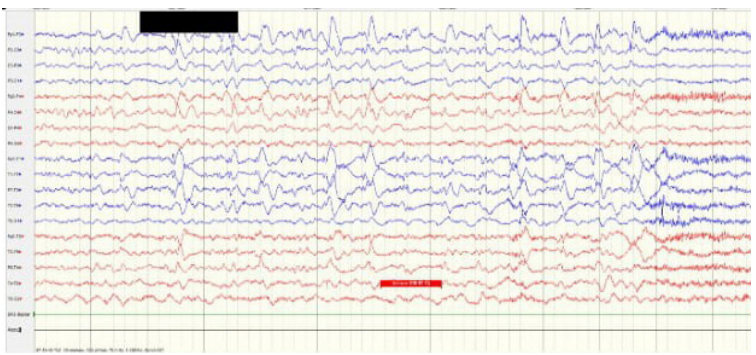

D

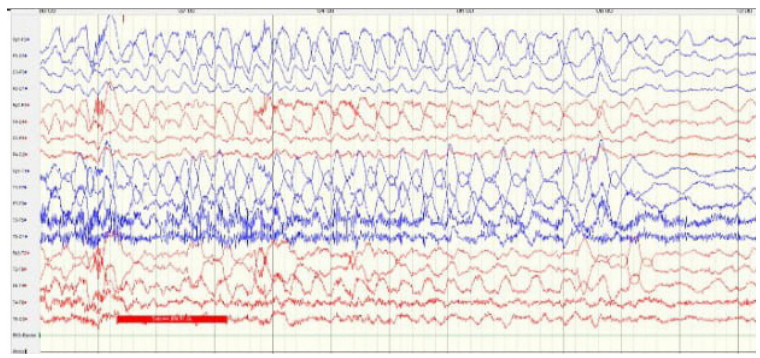

C

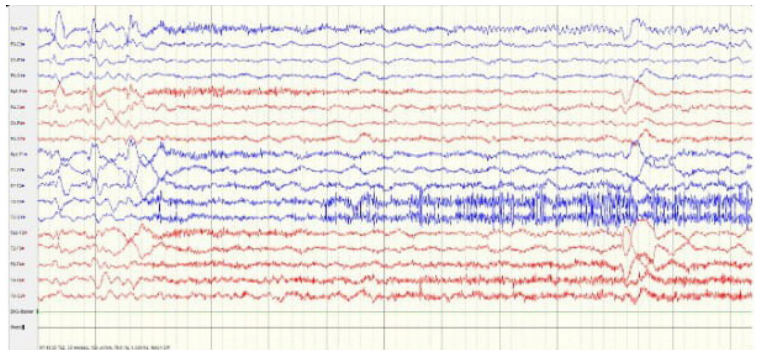

E

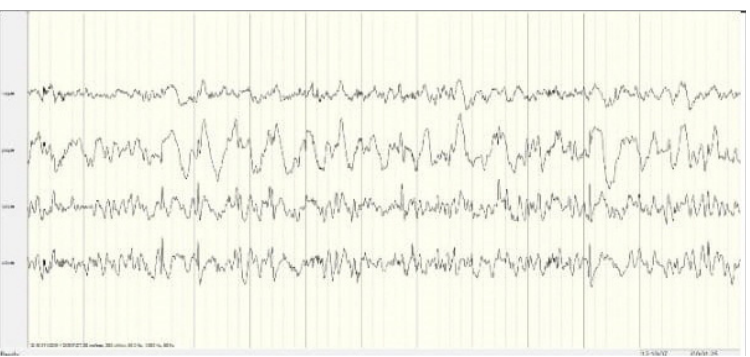

Figure 1. (A) Bipolar anteroposterior longitudinal montage: interictal spikes along with fast activity noted in the left frontal. (B) Preictal potentiation of sharp wave discharges. (C) Diffuse background attenuation at ictal onset. (D) Rhythmic spike and wave discharges noted in the left frontocentral region. (E) Four-contact electrocorticography shows repeated spiking in contact points 3 and 4.
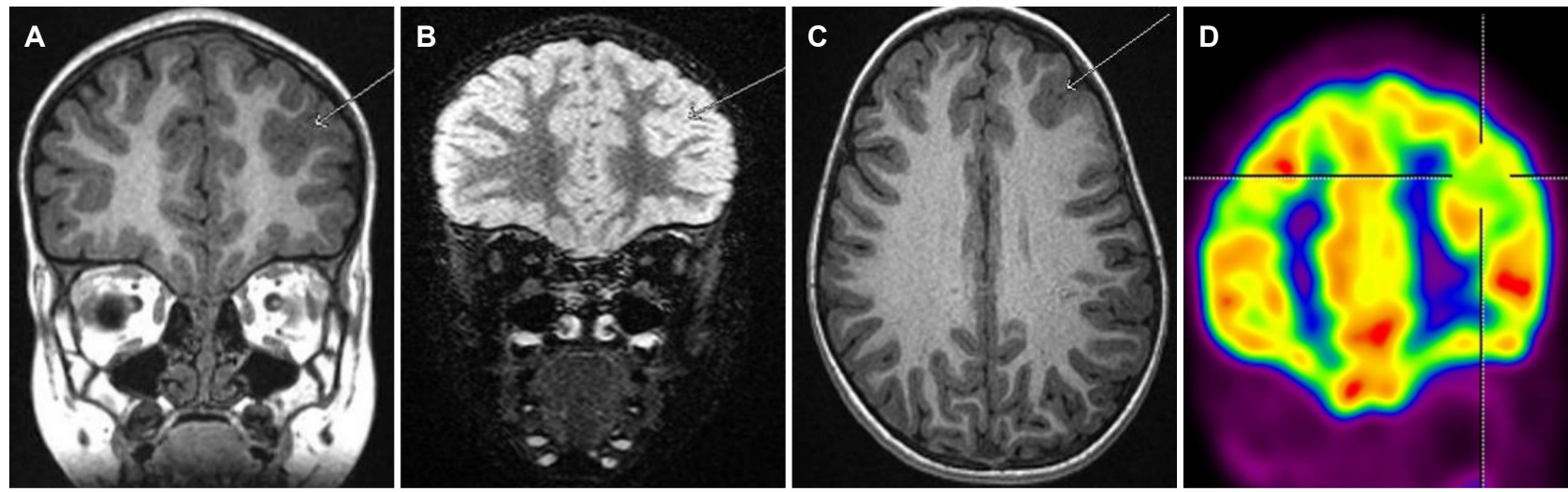

Figure 2. Magnetic resonance imaging showed abnormal gyral pattern with cortical thickening and blurring of grey-white matter junction in the left middle frontal gyrus (A-C) and interictal fluorodeoxyglucose positron emission tomography (coronal view) revealed corresponding frontal hypometabolism (D). 
medications (phenytoin [8 mg/kg/day], phenobarbitone [8 mg/kg/day], perampenel [2 $\mathrm{mg} /$ day], oxcarbazepine [30 mg/kg/day], topiramate [5 mg/kg/day], zonisamide [5 mg/kg/day] and clobazam [15 mg/day]). A 5-day course of methylprednisolone was given after obtaining cerebrospinal fluid for analysis. The latter showed protein of $17 \mathrm{mg} \%$, sugar $65 \mathrm{mg} \%$ (corresponding random blood sugar - $100 \mathrm{mg} \%$ ), no cells, normal immunoglobulin $\mathrm{G}$ index, and a negative gram stain. Serum and cerebrospinal fluid comprehensive autoimmune encephalitis panel (NMDA, GABAR, LGI1, CASPR2) was negative.

As the patient did not respond to anti-epileptic drugs (AEDs) and immunomodulation, a detailed discussion was done by the epilepsy surgery team with the parents for an early surgical resection of FCD. Presurgical development assessment was done using Griffith Mental Development scale and her development quotient (DQ) was $>85$ (normal). The challenges faced were the poor response to AEDs and immunomodulation and the minimal literature available on the very early surgery. Under AXIEM (Medtronic Stealth station S7) neuronavigation craniotomy was planned to centre over the lesion. A left frontal craniotomy was done to visualise the dysplastic middle frontal gyrus. The gyral pattern in the region appeared as puckered with the dysplastic gyri falling into a sulcus which extended deep within. ECoG performed using four channel strip electrode revealed spikes maximally from the margins of the lesion. Also, a depth electrode placed into the sulcus showed spikes well localising the area for resection. A complete excision of the lesion was done going along the margins of the dysplastic, thickened, greyish gyri which extended towards the depth of the sulcus. Post resection ECOG from the margins and walls of the resection cavity showed complete silence of the spikes which were recorded before resection (Fig. 1). Child remains seizure free till date (32 months) and is off anti-epileptic medications for the past 3 months. Post-surgical cognitive assessment after one year was normal (DQ>85). Histopathologic examination and immunohistochemistry of resected tissue revealed features consistent with focal cortical dysplasia type II B.

\section{Discussion}

Focal cortical dysplasia is the most common cause of refractory focal epilepsy and surgical resection is the only hope of seizure freedom for these patients with this type of epilepsy. The present case posed a challenge in terms of decision making as epilepsy surgery in the very early phase of refractory epilepsy is not an easy call to make neither for the epilepsy team nor for the patient's family. The unequivocal superiority of surgery over medical management constrained us to choose it over an uncertain waiting game where the patient is passively subjected to the flurry of seizures every day. ${ }^{1-3}$

The clinical semiology was analysed for the pattern of seizure spread, which suggested the involvement of premotor-posterior prefrontal pathways and medial spread to momentarily involve the cingulate cortex, which would explain a predominant elementary kind of automatism and subsequent laughter. ${ }^{4}$ Before the clinical onset of seizures, there was preictal potentiation of spikes for several seconds. This pattern has been recognized as a surrogate marker for type 2 FCD. ${ }^{5}$ This along with the presence of fast rhythmic activity interspersed with spikes and fast spikes in the IED also enabled us to communicate to the parents about better prognosis after surgery. This asserts the importance of carefully extracting electrographic signatures of FCD in addition to localization of the seizure onset zone. The anatomo-electro-clinical hypothesis was established beyond doubt by non-invasive means while the final verdict on the epileptogenicity of the lesion and the evidence of normality for the post resective margins were provided by an ECOG using a four-contact depth and strip electrode. Tailored resection of FCD has been found to be safe and effective in paediatric patients. ${ }^{6}$

The presence of type II FCD along with definition and resection of the epileptogenic zone in its totality has been the two proven factors determining the surgical outcome. ${ }^{7,8}$ Some authors have also found that the presence of a lesion on MRI was independently associated with a better outcome. This could be because of the fact that the MR positive cases are usually FCD type II. ${ }^{9}$ Nearly $90 \%$ of FCD type II b resection has been invariably associated with Engel class I outcome. ${ }^{9,10}$ The average time period from clinical onset to successful surgical resection in FCD is about 5-10 years. Even in the largest case series by Wang et al. ${ }^{11}$ that has around 112 children with FCD type II, the mean duration between seizure onset and surgery was 2.3 years (3.6 months-14.3 years). Early surgery has better developmental outcome as supported by Fauser et al. ${ }^{12}$ In the case scenario presented, the presence of a surgically resectable well-delineated lesion with electro-clinical correlation justified early surgery and that had an impact on better developmental outcome.

\section{Conflict of Interest}

The authors declare that they have no conflicts of interest. 


\section{Acknowledgements}

Dr Hamza -Director: ASTER MIMS, Kozhikode.

Dr Farhan Yasir-CEO: ASTER MIMS, Kozhikode.

\section{References}

1. Engel J Jr, McDermott MP, Wiebe $S$, et al. Early surgical therapy for drug-resistant temporal lobe epilepsy: a randomized trial. JAMA 2012; 307:922-30.

2. Wiebe S, Blume WT, Girvin JP, Eliasziw M; Effectiveness and Efficiency of Surgery for Temporal Lobe Epilepsy Study Group. A randomized, controlled trial of surgery for temporal-lobe epilepsy. N Eng/ J Med 2001;345:311-8.

3. Dwivedi R, Ramanujam B, Chandra PS, et al. Surgery for drug-resistant epilepsy in children. N Eng/ J Med 2017;377:1639-47.

4. Bonini F, McGonigal A, Trébuchon A, et al. Frontal lobe seizures: from clinical semiology to localization. Epilepsia 2013;55:264-77.

5. Lagarde S, Buzori S, Trebuchon A, et al. The repertoire of seizure onset patterns in human focal epilepsies: determinants and prognostic values. Epilepsia 2019;60:85-95.
6. Terra VC, Thomé U, Rosset SS, et al. Surgery for focal cortical dysplasia in children using intraoperative mapping. Childs Nerv Syst 2014;30: 1839-51.

7. Rowland NC, Englot DJ, Cage TA, Sughrue ME, Barbaro NM, Chang EF. A meta-analysis of predictors of seizure freedom in the surgical management of focal cortical dysplasia. J Neurosurg 2012;116:1035-41.

8. Choi SA, Kim SY, Kim H, et al. Surgical outcome and predictive factors of epilepsy surgery in pediatric isolated focal cortical dysplasia. Epilepsy Res 2018;139:54-9.

9. Isler C, Kucukyuruk B, Ozkara C, et al. Comparison of clinical features and surgical outcome in focal cortical dysplasia type 1 and type 2. Epilepsy Res 2017;136:130-6.

10. Chen HH, Chen C, Hung SC, et al. Cognitive and epilepsy outcomes after epilepsy surgery caused by focal cortical dysplasia in children: early intervention maybe better. Childs Nerv Syst 2014;30:1885-95.

11. Wang TS, Liu QZ, Liu M, et al. Clinical features and surgical outcomes in young children with focal cortical dysplasia type II. CNS Neurosci Ther 2020;26:270-7.

12. Fauser $S$, Essang $C$, Altenmüller DM, et al. Long-term seizure outcome in 211 patients with focal cortical dysplasia. Epilepsia 2015;56:66-76. 